\title{
Non-symmetric discrete Toda systems from quad-graphs
}

\author{
Raphael Boll ${ }^{1}$ and Yuri B. Suris ${ }^{2}$
}

November 21, 2018

\begin{abstract}
For all non-symmetric discrete relativistic Toda type equations we establish a relation to $3 \mathrm{D}$ consistent systems of quad-equations. Unlike the more simple and better understood symmetric case, here the three coordinate planes of $\mathbb{Z}^{3}$ carry different equations. Our construction allows for an algorithmic derivation of the zero curvature representations and yields analogous results also for the continuous time case.
\end{abstract}

\section{Introduction}

This paper is devoted to one aspect of the general topic of discrete integrable systems. In the recent years the viewpoint that discrete integrable systems are in a sense more fundamental than continuous ones becomes gradually more and more accepted in the soliton theory community. The last developments led to the understanding that the very definition of integrability becomes more transparent and natural on the discrete level, see [11. One can consider certain types of discrete equations, like the so called quadequations, as a sort of "elementary particles" of the solitonic world, with the enormous richness of this world resulting from various combinations and limiting procedures of these elementary objects. Here, we discover such "elementary particles" underlying the so called equations of the relativistic Toda type. This "microstructure" become visible only after the discretization procedure, and even then some hidden degrees of freedom remain to be uncovered in order for the whole simplicity to become apparent.

We review the relativistic Toda type equations and their integrable discretizations in Sections 2 and 3, respectively. After that, general Toda equations on graphs are discussed in Section 4. Fundamental notion of quad-graphs and quad-equations (which belong, in our view, to the "elementary particles" mentioned above) are discussed in Section 5. while the following Section [6 is devoted to the explanation of how the Toda type equations on graphs can be reduced to the systems of quad-equations. In Section 7 some combinatorial aspects of the regular triangular lattice are discussed, since this lattice underlies the discrete relativistic Toda type equations. Finally, Sections 810 contain

\footnotetext{
${ }^{1}$ Zentrum Mathematik, Technische Universität München, Boltzmannstr. 3, 85748 Garching, Germany

${ }^{2}$ Institut für Mathematik, MA 7-2, Technische Universität Berlin, Str. des 17. Juni 136, 10623 Berlin, Germany
} 
the main new results of this paper. Namely, in Section 8 we present the systems of quadequations which yield the non-symmetric discrete relativistic Toda equations. In Section

9 we show that all this systems have their common origin in just one master system of this type. In Section 10 we demonstrate how to derive the zero curvature representations for relativistic Toda type equations (discrete and continuous) in an algorithmic manner. A brief outlook is formulated in the concluding Section [11.

\section{Lattice equations of the relativistic Toda type}

The term "equations of the relativistic Toda type" is used to denote integrable lattice equations of the general form

$$
\begin{gathered}
\ddot{x}_{k}=r\left(\dot{x}_{k}\right)\left(\dot{x}_{k+1} f\left(x_{k+1}-x_{k}\right)-\dot{x}_{k-1} f\left(x_{k}-x_{k-1}\right)\right. \\
\left.+g\left(x_{k+1}-x_{k}\right)-g\left(x_{k}-x_{k-1}\right)\right) .
\end{gathered}
$$

The relativistic Toda lattice proper was invented by S. Ruijsenaars [14]. It is described by Newtonian equations of motion

$$
\begin{aligned}
& \ddot{x}_{k}=\left(1+\alpha \dot{x}_{k+1}\right)\left(1+\alpha \dot{x}_{k}\right) \frac{e^{x_{k+1}-x_{k}}}{1+\alpha^{2} e^{x_{k+1}-x_{k}}} \\
&-\left(1+\alpha \dot{x}_{k}\right)\left(1+\alpha \dot{x}_{k-1}\right) \frac{e^{x_{k}-x_{k-1}}}{1+\alpha^{2} e^{x_{k}-x_{k-1}}} .
\end{aligned}
$$

Here $\alpha$ is a small parameter whose physical meaning is the inverse speed of light. In the non-relativistic limit $\alpha \rightarrow 0$ system (2) turns into the usual Toda lattice.

It took about a decade for further integrable equations of the relativistic Toda type to be discovered. In [16] the following ones were found: two systems which again can be considered as $\alpha$-perturbations of the usual Toda lattice:

$$
\begin{aligned}
\ddot{x}_{k}= & \left(1+\alpha \dot{x}_{k+1}\right) e^{x_{k+1}-x_{k}}-\left(1+\alpha \dot{x}_{k-1}\right) e^{x_{k}-x_{k-1}} \\
& -\alpha^{2} e^{2\left(x_{k+1}-x_{k}\right)}+\alpha^{2} e^{2\left(x_{k}-x_{k-1}\right)}
\end{aligned}
$$

and

$$
\ddot{x}_{k}=\left(1-\alpha \dot{x}_{k}\right)^{2}\left(\left(1-\alpha \dot{x}_{k+1}\right) e^{x_{k+1}-x_{k}}-\left(1-\alpha \dot{x}_{k-1}\right) e^{x_{k}-x_{k-1}}\right),
$$

and two systems which can be considered as $\alpha$-perturbations of the so called modified Toda lattice:

$$
\begin{aligned}
\ddot{x}_{k}= & \dot{x}_{k}\left(e^{x_{k+1}-x_{k}}-e^{x_{k}-x_{k-1}}\right) \\
& +\alpha\left(\dot{x}_{k+1} \dot{x}_{k} \frac{e^{x_{k+1}-x_{k}}}{1+\alpha e^{x_{k+1}-x_{k}}}-\dot{x}_{k} \dot{x}_{k-1} \frac{e^{x_{k}-x_{k-1}}}{1+\alpha e^{x_{k}-x_{k-1}}}\right)
\end{aligned}
$$


and

$$
\ddot{x}_{k}=\dot{x}_{k}\left(1-\alpha \dot{x}_{k}\right)\left(\left(1-\alpha \dot{x}_{k+1}\right) \frac{e^{x_{k+1}-x_{k}}}{1+\alpha e^{x_{k+1}-x_{k}}}-\left(1-\alpha \dot{x}_{k-1}\right) \frac{e^{x_{k}-x_{k-1}}}{1+\alpha e^{x_{k}-x_{k-1}}}\right) .
$$

For all these systems a Lagrangian and a Hamiltonian formulations were given, and their complete integrability was demonstrated by presenting the full set of integrals of motion and a local zero curvature representations of the type

$$
\dot{L}_{k}=M_{k+1} L_{k}-L_{k} M_{k}
$$

in terms of $2 \times 2$ matrices (locality means that the matrix $L_{k}$ depends only on $x_{k}$ and the corresponding canonically conjugate momentum $p_{k}$, and not on phase variables from other lattice sites). Two further systems of the relativistic Toda type with rational interactions (as opposed to exponential interactions in the previous ones) appeared in [17:

$$
\ddot{x}_{k}=\dot{x}_{k}\left(x_{k+1}-2 x_{k}+x_{k-1}\right)+\frac{\alpha \dot{x}_{k+1} \dot{x}_{k}}{1+\alpha\left(x_{k+1}-x_{k}\right)}-\frac{\alpha \dot{x}_{k} \dot{x}_{k-1}}{1+\alpha\left(x_{k}-x_{k-1}\right)}
$$

and

$$
\ddot{x}_{k}=\dot{x}_{k}\left(1+\alpha^{2} \dot{x}_{k}\right)\left(\frac{x_{k+1}-x_{k}-\alpha \dot{x}_{k+1}}{1+\alpha\left(x_{k+1}-x_{k}\right)}-\frac{x_{k}-x_{k-1}-\alpha \dot{x}_{k-1}}{1+\alpha\left(x_{k}-x_{k-1}\right)}\right) .
$$

Both these systems are $\alpha$-perturbations of the so-called dual Toda lattice. Also for these systems the Lagrangian and the Hamiltonian formulations were given in [17], as well as a demonstration of their complete integrability. However, local zero curvature representations were not given at that point.

Along another line of research, a complete classification of "integrable" systems of the type (10) was achieved in [6]. The notion of "integrability" used in this paper is a clever and unexpected device, allowing to carry out a complete classification, but it has, à priori, nothing to do with the usual Liouville-Arnold integrability. Namely, they noticed that the above systems are always Lagrangian, and required that their form be retained under a sort of Legendre transformation. This allowed them to find all the Newtonian equations mentioned above, as well a new series of systems, including the most general one,

$$
\begin{gathered}
\ddot{x}_{k}=-\frac{1}{2}\left(\dot{x}_{k}^{2}-\nu^{2}\right)\left(\frac{\sinh 2\left(x_{k+1}-x_{k}\right)-\nu^{-1} \sinh (2 \nu \alpha) \dot{x}_{k+1}}{\sinh ^{2}\left(x_{k+1}-x_{k}\right)-\sinh ^{2}(\nu \alpha)}-\right. \\
\left.-\frac{\sinh 2\left(x_{k}-x_{k-1}\right)-\nu^{-1} \sinh (2 \nu \alpha) \dot{x}_{k-1}}{\sinh ^{2}\left(x_{k}-x_{k-1}\right)-\sinh ^{2}(\nu \alpha)}\right)
\end{gathered}
$$

its limiting case (first rescale $x_{k} \mapsto \nu x_{k}, \nu \mapsto \gamma \nu$, and then send $\nu \rightarrow 0$ ):

$$
\ddot{x}_{k}=-\left(\dot{x}_{k}^{2}-\gamma^{2}\right)\left(\frac{x_{k+1}-x_{k}-\alpha \dot{x}_{k+1}}{\left(x_{k+1}-x_{k}\right)^{2}-\gamma^{2} \alpha^{2}}-\frac{x_{k}-x_{k-1}-\alpha \dot{x}_{k-1}}{\left(x_{k}-x_{k-1}\right)^{2}-\gamma^{2} \alpha^{2}}\right),
$$


as well as the particular cases $\nu=0$, resp. $\gamma=0$, of the latter two systems:

$$
\begin{aligned}
\ddot{x}_{k}=-\dot{x}_{k}^{2} & \left(\operatorname{coth}\left(x_{k+1}-x_{k}\right)-\operatorname{coth}\left(x_{k}-x_{k-1}\right)\right. \\
& \left.-\frac{\alpha \dot{x}_{k+1}}{\sinh ^{2}\left(x_{k+1}-x_{k}\right)}+\frac{\alpha \dot{x}_{k-1}}{\sinh ^{2}\left(x_{k}-x_{k-1}\right)}\right)
\end{aligned}
$$

and

$$
\ddot{x}_{k}=-\dot{x}_{k}^{2}\left(\frac{1}{x_{k+1}-x_{k}}-\frac{1}{x_{k}-x_{k-1}}-\frac{\alpha \dot{x}_{k+1}}{\left(x_{k+1}-x_{k}\right)^{2}}+\frac{\alpha \dot{x}_{k-1}}{\left(x_{k}-x_{k-1}\right)^{2}}\right) .
$$

Complete integrability of these systems in the usual sense, along with $2 \times 2$ local zero curvature representations for the last two ones, was demonstrated in the monograph [18].

\section{Time discretization of the relativistic Toda type equa- tions}

Integrable discretizations of the relativistic Toda type equations all have the following general shape of discrete time Newtonian equations of motion:

$$
\begin{aligned}
& F\left(\widetilde{x}_{k}-x_{k}\right)-F(x_{k}-\underbrace{}_{x})= \\
& \quad G\left(x_{k+1}-x_{k}\right)-G\left(x_{k}-x_{k-1}\right)+H(\underbrace{x}_{k+1}-x_{k})-H\left(x_{k}-\widetilde{x}_{k-1}\right) .
\end{aligned}
$$

Here and below we use the following abbreviations for functions of the discrete time $h \mathbb{Z}$ :

$$
x_{k}=x_{k}(t), \quad \widetilde{x}_{k}=x_{k}(t+h), \quad x_{k}=x_{k}(t-h) .
$$

The integrability preserving time discretization of the Ruijsenaars' relativistic Toda lattice was performed in [15], where the following equations were derived:

$$
\frac{1+\alpha h^{-1}\left(e^{\widetilde{x}_{k}-x_{k}}-1\right)}{1+\alpha h^{-1}\left(e^{x_{k}-x_{k}}-1\right)}=\frac{\left(1+\alpha^{2} e^{x_{k+1}-x_{k}}\right)\left(1+\alpha(\alpha-h) e^{x_{k}-\widetilde{x}_{k-1}}\right)}{\left(1+\alpha^{2} e^{x_{k}-x_{k-1}}\right)\left(1+\alpha(\alpha-h) e^{x_{k+1}-x_{k}}\right)} .
$$

It was shown in [15] that, upon a natural Lagrangian (or discrete Hamiltonian) reformulation in terms of the canonically conjugate variables $x_{k}, p_{k}$, discrete time equations (15) share integrals of motion with the continuous time equations (2), and therefore have the same integrability properties (belong to the same integrable hierarchy). This remains true also for all integrable discretizations in this section.

The Newtonian systems (3) and (4) were discretized in [16] in an "additive" manner as

$$
\begin{aligned}
e^{\widetilde{x}_{k}-x_{k}}-e^{x_{k}-x_{k}}= & h \alpha e^{x_{k+1}-x_{k}}-h \alpha e^{x_{k}-x_{k-1}} \\
& -\frac{h(\alpha-h) e^{x_{k+1}-x_{k}}}{1-h \alpha e^{x_{k+1}-x_{k}}}+\frac{h(\alpha-h) e^{x_{k}-\widetilde{x}_{k-1}}}{1-h \alpha e^{x_{k}-\widetilde{x}_{k-1}}}
\end{aligned}
$$


and

$$
\begin{gathered}
\frac{1}{1-\alpha h^{-1}\left(e^{\left.\widetilde{x}_{k}-x_{k}-1\right)}\right.}-\frac{1}{1-\alpha h^{-1}\left(e^{x_{k}-x_{k}}-1\right)}= \\
=\alpha(\alpha+h) e^{x_{k+1}^{x_{k}-x_{k}}-\alpha(\alpha+h) e^{x_{k}-\widetilde{x}_{k-1}}-\alpha^{2} e^{x_{k+1}-x_{k}}+\alpha^{2} e^{x_{k}-x_{k-1},},}
\end{gathered}
$$

while the discretizations of the systems (5) and (6) given there were "multiplicative":

$$
\frac{\left(e^{\widetilde{x}_{k}-x_{k}}-1\right)}{\left(e^{x_{k}-x_{k}}-1\right)}=\frac{\left(1+h e^{x_{k+1}-x_{k}}\right)\left(1+\alpha e^{x_{k}-\widetilde{x}_{k-1}}\right)\left(1+\alpha e^{x_{k+1}-x_{k}}\right)}{\left(1+\alpha e^{x_{k+1}-x_{k}}\right)\left(1+h e^{x_{k}-\widetilde{x}_{k-1}}\right)\left(1+\alpha e^{x_{k}-x_{k-1}}\right)}
$$

and

$$
\begin{aligned}
& \frac{\left(e^{\widetilde{x}_{k}-x_{k}}-1\right)}{\left(e^{x_{k}-x_{k}}-1\right)} \cdot \frac{1-\alpha h^{-1}\left(e^{x_{k}-x_{k}}-1\right)}{1-\alpha h^{-1}\left(e^{\left.\widetilde{x}_{k}-x_{k}-1\right)}\right.}= \\
& \quad=\frac{\left(1+\alpha e^{x_{k}-x_{k-1}}\right)}{\left(1+\alpha e^{x_{k+1}-x_{k}}\right)} \cdot \frac{\left(1+(\alpha+h) e^{x_{k+1}-x_{k}}\right)}{\left(1+(\alpha+h) e^{x_{k}-\widetilde{x}_{k-1}}\right)}
\end{aligned}
$$

Discretizations of the rational systems (8) and (9) appeared in [17]:

$$
\frac{\widetilde{x}_{k}-x_{k}}{x_{k}-\underline{x}_{k}}=\frac{\left(1+h(\underbrace{}_{k+1}-x_{k})\right)}{\left(1+\alpha\left(x_{k+1}-x_{k}\right)\right)} \cdot \frac{\left(1+\alpha\left(x_{k}-\widetilde{x}_{k-1}\right)\right)}{\left(1+h\left(x_{k}-\widetilde{x}_{k-1}\right)\right)} \cdot \frac{\left(1+\alpha\left(x_{k+1}-x_{k}\right)\right)}{\left(1+\alpha\left(x_{k}-x_{k-1}\right)\right)}
$$

and

$$
\begin{aligned}
& \frac{\left(\widetilde{x}_{k}-x_{k}\right)\left(1+\alpha(\alpha+h) h^{-1}\left(x_{k}-x_{k}\right)\right)}{(x_{k}-\underbrace{}_{k})\left(1+\alpha(\alpha+h) h^{-1}\left(\widetilde{x}_{k}-x_{k}\right)\right)}= \\
& =\frac{\left(1+\alpha\left(x_{k}-x_{k-1}\right)\right)}{\left(1+\alpha\left(x_{k+1}-x_{k}\right)\right)} \cdot \frac{\left(1+(\alpha+h)\left(x_{k+1}-x_{k}\right)\right)}{\left(1+(\alpha+h)\left(x_{k}-\widetilde{x}_{k-1}\right)\right)}
\end{aligned}
$$

A discrete version of the device from [6] was developed in [1]. It was used to classify "integrable" systems of the general type (14), i.e., those retaining their form under a sort of a discrete Legendre transformation. The resulting list consisted essentially of the systems quoted in above in this section, as well as of discretizations of the systems 
(10) -(13) . A discretization of (10) reads

$$
\begin{aligned}
& \frac{\sinh \left(\widetilde{x}_{k}-x_{k}+\nu h\right)}{\sinh \left(\widetilde{x}_{k}-x_{k}-\nu h\right)} \cdot \frac{\sinh \left(x_{k}-{\underset{\sim}{x}}_{k}-\nu h\right)}{\sinh \left(x_{k}-{\underset{\sim}{x}}_{k}+\nu h\right)} \\
& =\frac{\sinh \left(x_{k+1}-x_{k}+\nu \alpha\right)}{\sinh \left(x_{k+1}-x_{k}-\nu \alpha\right)} \cdot \frac{\sinh \left(x_{k}-x_{k-1}-\nu \alpha\right)}{\sinh \left(x_{k}-x_{k-1}+\nu \alpha\right)} \\
& \quad \times \frac{\sinh (\underbrace{x}_{k+1}-x_{k}-\nu \alpha+\nu h)}{\sinh (\underbrace{x}_{k+1}-x_{k}+\nu \alpha-\nu h)} \cdot \frac{\sinh \left(x_{k}-\widetilde{x}_{k-1}+\nu \alpha-\nu h\right)}{\sinh \left(x_{k}-\widetilde{x}_{k-1}-\nu \alpha+\nu h\right)} .
\end{aligned}
$$

Its rational version, which is a discretization of (11), reads

$$
\begin{gathered}
\frac{\left(\widetilde{x}_{k}-x_{k}+\gamma h\right)}{\left(\widetilde{x}_{k}-x_{k}-\gamma h\right)} \cdot \frac{\left(x_{k}-x_{k}-\gamma h\right)}{(x_{k}-\underbrace{}_{k}+\gamma h)}=\frac{\left(x_{k+1}-x_{k}+\gamma \alpha\right)}{\left(x_{k+1}-x_{k}-\gamma \alpha\right)} \cdot \frac{\left(x_{k}-x_{k-1}-\gamma \alpha\right)}{\left(x_{k}-x_{k-1}+\gamma \alpha\right)} \times \\
\times \frac{(\underbrace{x}_{k+1}-x_{k}-\gamma \alpha+\gamma h)}{(\underbrace{x}_{k+1}-x_{k}+\gamma \alpha-\gamma h)} \cdot \frac{\left(x_{k}-\widetilde{x}_{k-1}+\gamma \alpha-\gamma h\right)}{\left(x_{k}-\widetilde{x}_{k-1}-\gamma \alpha+\gamma h\right)} .
\end{gathered}
$$

Finally, the $\nu \rightarrow 0$, resp. the $\gamma \rightarrow 0$ limits of the latter two equations lead to additive ones:

$$
\begin{gathered}
h \operatorname{coth}\left(\widetilde{x}_{k}-x_{k}\right)-h \operatorname{coth}\left(x_{k}-{\underset{\sim}{x}}_{k}\right)=\alpha \operatorname{coth}\left(x_{k+1}-x_{k}\right)-\alpha \operatorname{coth}\left(x_{k}-x_{k-1}\right)- \\
-(\alpha-h) \operatorname{coth}(\underbrace{}_{k+1}-x_{k})+(\alpha-h) \operatorname{coth}\left(x_{k}-\widetilde{x}_{k-1}\right)
\end{gathered}
$$

and

$$
\frac{h}{\widetilde{x}_{k}-x_{k}}-\frac{h}{x_{k}-\underline{x}_{k}}=\frac{\alpha}{x_{k+1}-x_{k}}-\frac{\alpha}{x_{k}-x_{k-1}}-\frac{\alpha-h}{\underbrace{}_{k+1}-x_{k}}+\frac{\alpha-h}{x_{k}-\widetilde{x}_{k-1}},
$$

which discretize (12) and (13), respectively. The integrability of the difference equations (22) -(25) in the usual sense was dealt with in [18], where it was shown that they share the integrals of motion and the matrices $L_{k}$ from the zero curvature representations with their continuous time counterparts.

\section{Discrete Toda type equations on graphs}

An important observation made in [2] was that the natural combinatorial structure underlying the discrete relativistic Toda type lattices (14) is actually the regular triangular lattice in the plane (rather than the standard square lattice $\mathbb{Z}^{2}$ ). Namely, each equation of the system (14) relates seven fields assigned to the star of a vertex of the regular triangular lattice, each one of the functions $F, G, H$ being associated to edges of one of the tree directions, see Figure 1.

Note that while in the systems (15)-(21) (called hereafter non-symmetric discrete relativistic Toda type equations) the tree functions $F, G, H$ are, generally speaking, dif-

ferent, this is no more the case for systems (22)-(25). In the latter systems (called 


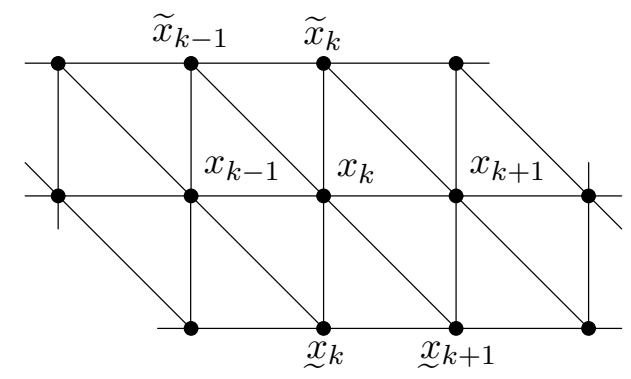

Figure 1: Regular triangular lattice underlying discrete relativistic Toda type systems

hereafter symmetric discrete relativistic Toda type equations) all three functions essentially coincide, differing only by the values of the built-in parameters.

This allows, at least for symmetric systems, the next generalization step, which was made in [3], namely the introduction of discrete Toda type systems on arbitrary graphs.

Definition 1. Let $\mathcal{G}$ be a graph, with the set of vertices $V(\mathcal{G})$ and the set of edges $E(\mathcal{G})$. A discrete Toda type system on $\mathcal{G}$ for a function $x: V(\mathcal{G}) \rightarrow \mathbb{C}$ reads:

$$
\sum_{v \in \operatorname{star}\left(v_{0}\right)} \phi\left(x_{0}, x\right)=0
$$

There is one equation for every vertex $v_{0} \in V(\mathcal{G})$; the summation is extended over $\operatorname{star}\left(v_{0}\right)$, the set of vertices of $\mathcal{G}$ connected to $v_{0}$ by an edge (see Figure 2); we write $x_{0}=x\left(v_{0}\right)$ and $x=x(v)$ and often suppress the notational difference between the vertices $v$ of the graph and the fields $x=x(v)$ assigned to them. Often, the function $\phi=\phi\left(x_{0}, x ; \alpha\right)$ is supposed to additionally depend on some parameters $\alpha: E(\mathcal{G}) \rightarrow \mathbb{C}$, assigned to the edges of $\mathcal{G}$.

The notion of integrability of discrete Toda type systems is not well established yet. We discuss here a definition based on the notion of the discrete zero curvature representation which works under an additional assumption about the graph $\mathcal{G}$. Namely, it has to come from a strongly regular polytopal cell decomposition of an oriented surface.

We consider, in somewhat more detail, the dual graph (cell decomposition) $\mathcal{G}^{*}$. Each $\mathfrak{e} \in E(\mathcal{G})$ separates two faces of $\mathcal{G}$, which in turn correspond to two vertices of $\mathcal{G}^{*}$. A path between these two vertices is then declared the edge $\mathfrak{e}^{*} \in E\left(\mathcal{G}^{*}\right)$ dual to $\mathfrak{e}$. If one assigns a direction to an edge $\mathfrak{e} \in E(\mathcal{G})$, then it will be assumed that the dual edge $\mathfrak{e}^{*} \in E\left(\mathcal{G}^{*}\right)$ is also directed, in a way consistent with the orientation of the underlying surface, namely so that the pair $\left(\mathfrak{e}, \mathfrak{e}^{*}\right)$ is positively oriented at its crossing point. This orientation convention implies that $\mathfrak{e}^{* *}=-\mathfrak{e}$. Finally, the faces of $\mathcal{G}^{*}$ are in a one-to-one correspondence with the vertices of $\mathcal{G}$ : if $x_{0} \in V(\mathcal{G})$, and $x_{1}, \ldots, x_{n} \in V(\mathcal{G})$ are its neighbors connected with $x_{0}$ by the edges $\mathfrak{e}_{1}=\left(x_{0}, x_{1}\right), \ldots, \mathfrak{e}_{n}=\left(x_{0}, x_{n}\right) \in E(\mathcal{G})$, then the face of $\mathcal{G}^{*}$ dual to $x_{0}$ is bounded by the dual edges $\mathfrak{e}_{1}^{*}=\left(y_{1}, y_{2}\right), \ldots, \mathfrak{e}_{n}^{*}=\left(y_{n}, y_{1}\right)$; see Figure 3 , 


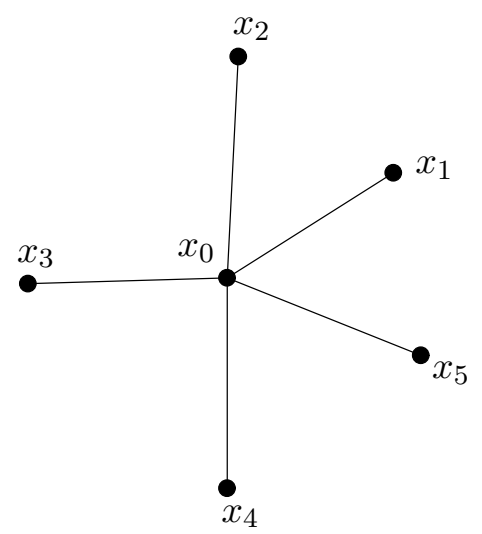

Figure 2: Star of a vertex $x_{0}$ in the graph $\mathcal{G}$.

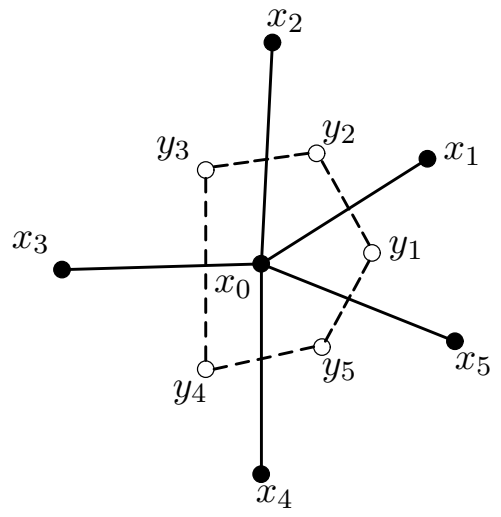

Figure 3: Face of $\mathcal{G}^{*}$ dual to a vertex $x_{0}$ of $\mathcal{G}$.

We will say that a discrete Toda type system on $\mathcal{G}$ possesses a discrete zero curvature representation if there is a collection of matrices $L\left(\mathfrak{e}^{*} ; \lambda\right) \in G[\lambda]$ from some loop group $G[\lambda]$, associated to directed edges $\mathfrak{e}^{*} \in \vec{E}\left(\mathcal{G}^{*}\right)$ of the dual graph $\mathcal{G}^{*}$, such that:

- the matrix $L\left(\mathfrak{e}^{*} ; \lambda\right)=L\left(x_{0}, x, \alpha ; \lambda\right)$ depends on the fields $x_{0}$ and $x$ at the vertices of the edge $\mathfrak{e}=\left(x_{0}, x\right) \in E(\mathcal{G})$, dual to the edge $\mathfrak{e}^{*} \in E\left(\mathcal{G}^{*}\right)$, as well as on the parameter $\alpha=\alpha(\mathfrak{e})$;

- for any directed edge $\mathfrak{e}^{*}=\left(y_{1}, y_{2}\right)$, if $-\mathfrak{e}=\left(y_{2}, y_{1}\right)$, then

$$
L(-\mathfrak{e}, \lambda)=(L(\mathfrak{e}, \lambda))^{-1}
$$

- for any closed path of directed edges

$$
\mathfrak{e}_{1}^{*}=\left(y_{1}, y_{2}\right), \quad \mathfrak{e}_{2}^{*}=\left(y_{2}, y_{3}\right), \quad \ldots, \quad \mathfrak{e}_{n}^{*}=\left(y_{n}, y_{1}\right),
$$

we have

$$
L\left(\mathfrak{e}_{n}^{*}, \lambda\right) \cdots L\left(\mathfrak{e}_{2}^{*}, \lambda\right) L\left(\mathfrak{e}_{1}^{*}, \lambda\right)=\mathbf{1} .
$$

The matrix $L\left(\mathfrak{e}^{*} ; \lambda\right)$ is interpreted as a transition matrix along the edge $\mathfrak{e}^{*} \in E\left(\mathcal{G}^{*}\right)$, that is, a transition across the edge $\mathfrak{e} \in E(\mathcal{G})$.

Under conditions (27), (28) one can define a wave function $\Psi: V\left(\mathcal{G}^{*}\right) \rightarrow G[\lambda]$ on the vertices of the dual graph $\mathcal{G}^{*}$, by the following requirement: for any directed edge $\mathfrak{e}^{*}=\left(y_{1}, y_{2}\right)$, the values of the wave functions at its ends must be connected via

$$
\Psi\left(y_{2}, \lambda\right)=L\left(\mathfrak{e}^{*}, \lambda\right) \Psi\left(y_{1}, \lambda\right)
$$

For an arbitrary graph, the analytical consequences of the zero curvature representation for a given collection of equations are not clear. However, in the case of regular graphs, like the square lattice or the regular triangular lattice, such a representation 
may be used to determine conserved quantities for suitably defined Cauchy problems, as well as to apply powerful analytical methods for finding concrete solutions.

It was shown in [3] that discrete Toda type systems with the following functions $\phi$ are integrable in the above sense:

$$
\begin{aligned}
\phi\left(x_{0}, x ; \alpha\right) & =\frac{\alpha}{x-x_{0}}, \\
\phi\left(x_{0}, x ; \alpha\right) & =\alpha \operatorname{coth}\left(x-x_{0}\right) \\
\phi\left(x_{0}, x ; \alpha\right) & =\log \frac{x-x_{0}+\alpha}{x-x_{0}-\alpha} \\
\phi\left(x_{0}, x ; \alpha\right) & =\log \frac{\sinh \left(x-x_{0}+\alpha\right)}{\sinh \left(x-x_{0}-\alpha\right)} .
\end{aligned}
$$

See [3] for details about the admissible assignments of edge parameters $\alpha$. Actually the discrete Toda system with the functions (31) is reduced to the one with the functions (30) via the change of variables $x \mapsto \exp (2 x)$ and therefore actually does not need to be considered separately.

\section{Quad-graphs and quad-equations}

Although one can consider 2D integrable systems on very different kinds of graphs on surfaces, there is one kind - quad-graphs - supporting the most fundamental integrable systems.

Definition 2. A quad-graph $\mathcal{D}$ is a strongly regular polytopal cell decomposition of a surface with all quadrilateral faces.

Quad-graphs are privileged because from an arbitrary strongly regular polytopal cell decomposition $\mathcal{G}$ one can produce a certain quad-graph $\mathcal{D}$, called the double of $\mathcal{G}$. The double $\mathcal{D}$ is a quad-graph, constructed from $\mathcal{G}$ and its dual $\mathcal{G}^{*}$ as follows. The set of vertices of the double $\mathcal{D}$ is $V(\mathcal{D})=V(\mathcal{G}) \sqcup V\left(\mathcal{G}^{*}\right)$. Each pair of dual edges, say $\mathfrak{e}=\left(x_{0}, x_{1}\right) \in E(\mathcal{G})$ and $\mathfrak{e}^{*}=\left(y_{1}, y_{2}\right) \in E\left(\mathcal{G}^{*}\right)$, defines a quadrilateral $\left(x_{0}, y_{1}, x_{1}, y_{2}\right)$. These quadrilaterals constitute the faces of a cell decomposition (quad-graph) $\mathcal{D}$. Thus, a star of a vertex $x_{0} \in V(\mathcal{G})$ generates a flower of adjacent quadrilaterals from $F(\mathcal{D})$ around $x_{0}$; see Figure 4 . Let us stress that edges of $\mathcal{D}$ belong neither to $E(\mathcal{G})$ nor to $E\left(\mathcal{G}^{*}\right)$.

Quad-graphs $\mathcal{D}$ coming as doubles are bipartite: the set $V(\mathcal{D})$ may be decomposed into two complementary halves, $V(\mathcal{D})=V(\mathcal{G}) \sqcup V\left(\mathcal{G}^{*}\right)$ ("black" and "white" vertices), such that the ends of each edge from $E(\mathcal{D})$ are of different colors. Equivalently, any closed loop consisting of edges of $\mathcal{D}$ has an even length.

The construction of the double can be reversed. Start with a bipartite quad-graph $\mathcal{D}$. For instance, any quad-graph embedded in a plane or in an open disc is automatically bipartite. Any bipartite quad-graph produces two dual polytopal (in general, no more quadrilateral) cell decompositions $\mathcal{G}$ and $\mathcal{G}^{*}$, with $V(\mathcal{G})$ containing all the "black" vertices of $\mathcal{D}$ and $V\left(\mathcal{G}^{*}\right)$ containing all the "white" ones, and edges of $\mathcal{G}$ (resp. of $\mathcal{G}^{*}$ ) 


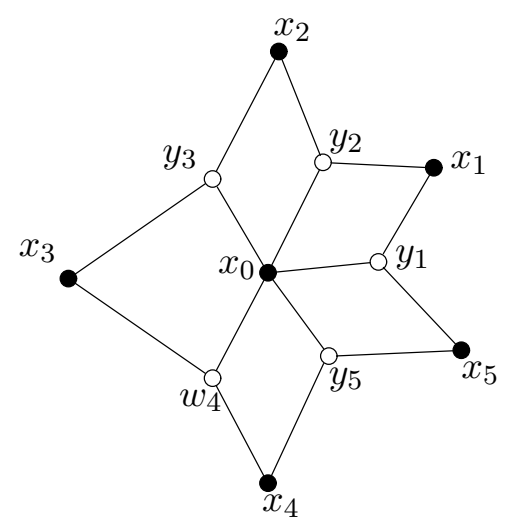

Figure 4: Faces of $\mathcal{D}$ around the vertex $x_{0}$.

connecting "black" (resp. "white") vertices along the diagonals of each face of $\mathcal{D}$. The decomposition of $V(\mathcal{D})$ into $V(\mathcal{G})$ and $V\left(\mathcal{G}^{*}\right)$ is unique, up to interchanging the roles of $\mathcal{G}$ and $\mathcal{G}^{*}$.

A privileged role played by the quad-graphs is reflected in the privileged role played in the theory of discrete integrable systems by the so called quad-equations supported by quad-graphs.

Definition 3. For a given bipartite quad-graph $\mathcal{D}$, the system of quad-equations for a function $x: V(\mathcal{D}) \rightarrow \mathbb{C}$ consists of equations of the type

$$
Q\left(x_{0}, y_{1}, x_{1}, y_{2}\right)=0
$$

see Figure 5. There is one equation for every face $\left(x_{0}, y_{1}, x_{1}, y_{2}\right)$ of $\mathcal{D}$. The function $Q$ is supposed to be multi-affine, i.e., a polynomial of degree $\leq 1$ in each argument, so that equation (34) is uniquely solvable for any of its arguments. Often, it is supposed that the function $Q=Q\left(x_{0}, y_{1}, x_{1}, y_{2} ; \alpha, \beta\right)$ additionally depends on some parameters usually assigned to the edges of the quadrilaterals, $\alpha: E(\mathcal{D}) \rightarrow \mathbb{C}$, so that the opposite edges carry equal parameters: $\alpha=\alpha\left(x_{0}, y_{1}\right)=\alpha\left(y_{2}, x_{1}\right)$ and $\beta=\alpha\left(x_{0}, y_{2}\right)=\alpha\left(y_{1}, x_{1}\right)$.

There exists a fundamental and surprisingly simple notion of 3D consistency of quadequations which can be put into the basis of the integrability theory, which has been done in [10] and [13. The property of 3D consistency allows one, in particular, to derive in an algorithmic way such basic integrability attributes as discrete zero curvature representations and Bäcklund transformations for quad-equations. Moreover, this property has been put [4] into the basis of a classification of integrable quad-equations which provided a finite list of such equations known nowadays as the "ABS list".

\section{From quad-equations to discrete Toda type systems}

The geometric relation of a given surface graph $\mathcal{G}$ to its double $\mathcal{D}$, described in Section 5. leads to a relation of discrete Toda type systems on $\mathcal{G}$ to quad-equations on $\mathcal{D}$. The 


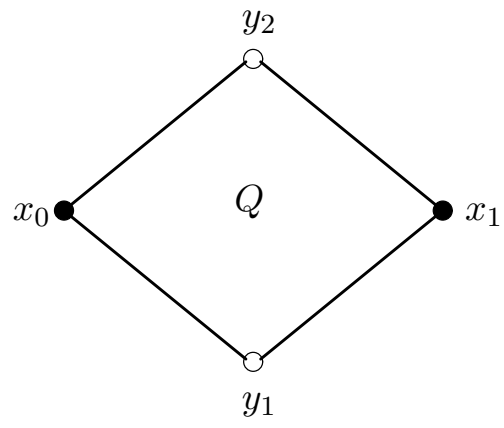

Figure 5: A quad-equation.

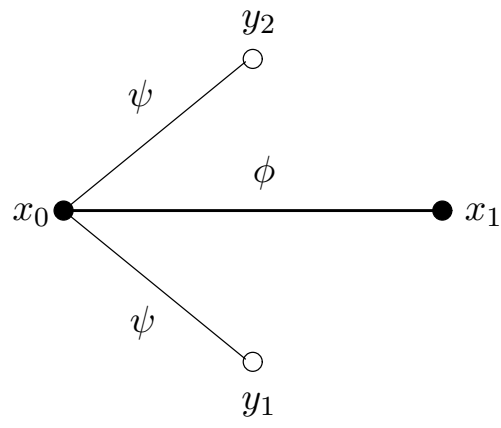

Figure 6: Three-leg form of a quad-equation.

latter relation is based on a deep and somewhat mysterious property of quad-equations which was discovered in several examples in [10, was established for all equations of the ABS list in [4, and was proved for all quad-equations with multi-affine functions $Q$ by V. Adler, see Exercise 6.16 in [1].

Definition 4. A quad-equation (34) possesses a three-leg form centered at the vertex $x_{0}$ if it is equivalent to the equation

$$
\psi\left(x_{0}, y_{1}\right)-\psi\left(x_{0}, y_{2}\right)=\phi\left(x_{0}, x_{1}\right)
$$

with some functions $\psi, \phi$. The terms on the left-hand side correspond to the "short" legs $\left(x_{0}, y_{1}\right),\left(x_{0}, y_{2}\right) \in E(\mathcal{D})$, while the right-hand side corresponds to the "long" leg $\left(x_{0}, x_{1}\right) \in E(\mathcal{G})$.

Summation of quad-graph equations for the flower of quadrilaterals adjacent to the "black" vertex $x_{0} \in V(\mathcal{G})$ (see Figure 4) immediately leads, due to the telescoping effect, to the following statement.

Theorem 1. a) Suppose that equation (34) on a bipartite quad-graph $\mathcal{D}$ possesses a three-leg form. Then the restriction of any solution $f: V(\mathcal{D}) \rightarrow \mathbb{C}$ to the "black" vertices $V(\mathcal{G})$ satisfies the discrete Toda type equations,

$$
\sum_{x_{k} \in \operatorname{star}\left(x_{0}\right)} \phi\left(x_{0}, x_{k}\right)=0 .
$$

b) Conversely, given a solution $f: V(\mathcal{G}) \rightarrow \mathbb{C}$ of the Toda type equations (36) on a simply connected surface graph $\mathcal{G}$, there exists a one-parameter family of extensions $f: V(\mathcal{D}) \rightarrow \mathbb{C}$ satisfying equation (34) on the double $\mathcal{D}$. Such an extension is uniquely determined by the value at one arbitrary vertex of $V\left(\mathcal{G}^{*}\right)$.

It was shown in [10] that symmetric discrete Toda type systems mentioned at the end of Section 4 come, through this construction, from the following integrable quadequations: the systems with legs (30) and (32) come from the $\delta=0$ and $\delta=1$ cases, respectively, of the so called Q1 equation of the ABS list, which reads

$$
\alpha\left(x_{0} y_{1}+x_{1} y_{2}\right)-\beta\left(x_{0} y_{2}+x_{1} y_{1}\right)-(\alpha-\beta)\left(x_{0} x_{1}+y_{1} y_{2}\right)+\delta \alpha \beta(\alpha-\beta)=0,
$$


while the system with legs (33) comes from the $\delta=0$ case of the so called Q3 equation of the ABS list, which reads

$$
\sinh (\alpha)\left(x_{0} y_{1}+x_{1} y_{2}\right)-\sinh (\beta)\left(x_{0} y_{2}+x_{1} y_{1}\right)-\sinh (\alpha-\beta)\left(x_{0} x_{1}+y_{1} y_{2}\right)=0 .
$$

\section{Triangular lattice}

In Section 6 we established, for symmetric discrete Toda systems on an arbitrary planar graph $\mathcal{G}$, a relation to integrable quad-equations on the double $\mathcal{D}$. For non-symmetric discrete relativistic Toda type systems, such a relation remained unknown until recently, and it constitutes the main new result of the present paper.

The non-symmetric discrete relativistic Toda type systems live on the regular triangular lattice $\mathcal{T}$ and cannot be directly generalized to arbitrary graphs. Therefore, we introduce now the specific notation tailored for the regular triangular lattice. The double of $\mathcal{T}$ is the quad-graph $\mathcal{K}$ known as the dual kagome lattice (drawn on Figure 7 in dashed lines). The latter graph has vertices of two kinds, black vertices of valence 6 and white vertices of valence 3 , and edges of three types, all edges of each type being parallel. The quadrilateral faces of the dual kagome lattice are of three different types. We will denote them by type I, II, and III, according to Figure 9 .

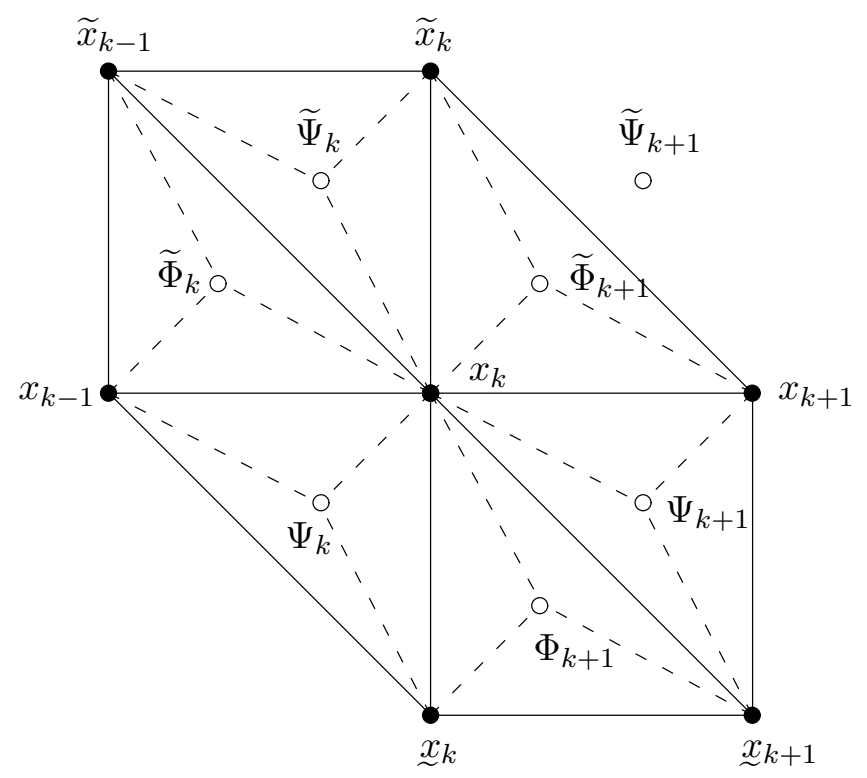

Figure 7: Fields and wave functions on the triangular lattice

The dual kagome lattice can be realized as a quad-surface in $\mathbb{Z}^{3}$, so that the three types of quadrilaterals are realized as elementary squares of $\mathbb{Z}^{3}$ parallel to the three coordinate planes (this is easy to see directly but follows also from the general theory of quasi-crystallic quad-graphs in [9]). In this realization, the black vertices of $\mathcal{K}$, that 
is, the vertices of $\mathcal{T}$, are the points $\left(i_{1}, i_{2}, i_{3}\right) \in \mathbb{Z}^{3}$ lying in the plane $i_{1}+i_{2}+i_{3}=0$, while the white vertices of $\mathcal{K}$ are the points of $\mathbb{Z}^{3}$ lying in the planes $i_{1}+i_{2}+i_{3}=1$ (the vertices $\Psi$ ) and $i_{1}+i_{2}+i_{3}=-1$ (the vertices $\Phi$ ). See Figure 8 .

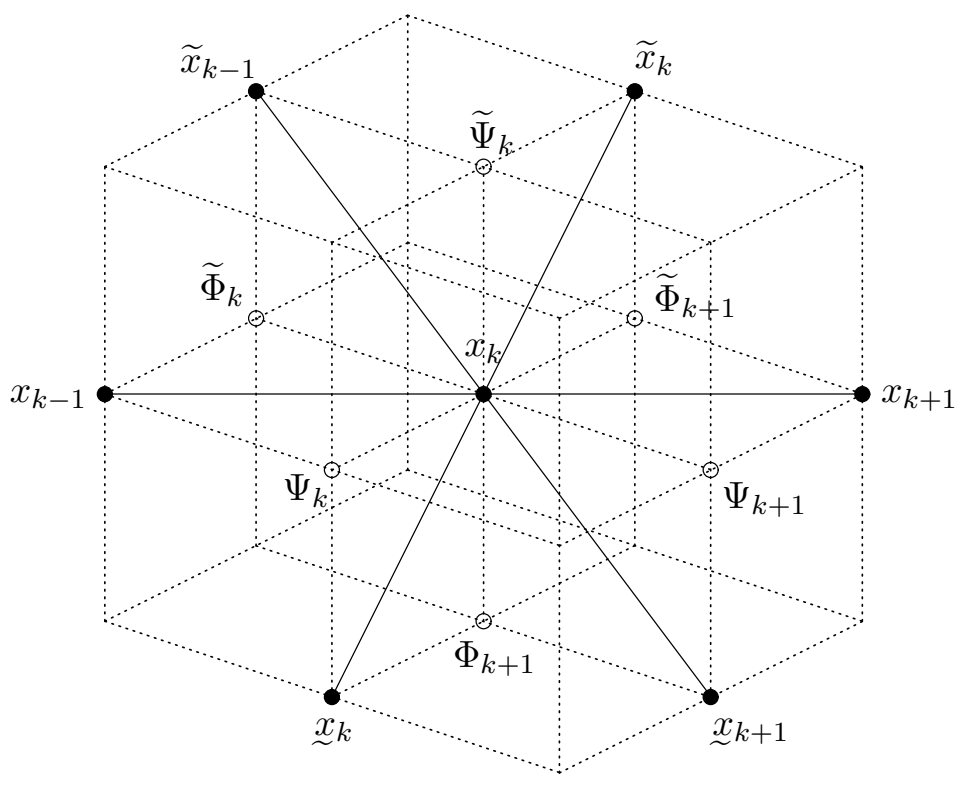

Figure 8: Embedding of the triangular lattice and the dual kagome lattice into $\mathbb{Z}^{3}$

\section{Discrete relativistic Toda type system from quad-equations on $\mathcal{K}$}

We now formulate the main result of the present paper.

Theorem 2. Each discrete relativistic Toda type system is a restriction to the triangular lattice $\mathcal{T}$ of a certain $3 D$ consistent system of quad-equations on the dual kagome lattice $\mathcal{K}$ considered as a quad-surface in $\mathbb{Z}^{3}$.

Proof of this theorem is obtained by a direct case-by-case construction of the corresponding systems of quad-equations (see, however, about the unifying "master system" in Section (9). For the lack of space, these systems are given below not for all discrete relativistic Toda systems, but for four of them only, namely, for (15), (16), (18), and (20). Details for other systems can be found in [12]. The systems are specified by giving the quad-equations explicitly for each type of quadrilateral faces separately in notation of Figure 9. One has to: a) find the three-leg forms, centered at $x_{k}$, of quad-equations for all six quadrilaterals around $x_{k}$ and then check that adding these three-leg forms results in the corresponding discrete Toda equation, and b) check the 3D consistency of the quad-equations. All this is a matter of direct computations which are easy enough 
to perform by hands but are better delegated to a symbolic manipulator like Maple or Mathematica.

System (15). 3D consistent system of quad-equations:

$$
\begin{aligned}
& \alpha h \lambda(X Y+U V)-\alpha h(\alpha-h) \lambda^{2} X U-\left((\alpha-h) \lambda^{2}+h\right) X V+\alpha \lambda^{2} Y V=0, \\
& \alpha \lambda(X Y+U V)-X V+\lambda^{2} Y V=0, \\
& \alpha(\alpha-h) \lambda(X Y+U V)+\alpha h(\alpha-h) X U+\left((\alpha-h) \lambda^{2}+h\right) X V-\alpha Y V=0 .
\end{aligned}
$$

The three-leg forms of these equations (centered at $x_{k}$ ) read:

$$
\begin{aligned}
& \left(\alpha e^{\widetilde{x}_{k}-x_{k}}-(\alpha-h)\right) \cdot \frac{h e^{x_{k}}+\lambda \widetilde{\Psi}_{k}}{\widetilde{\Psi}_{k}-(\alpha-h) \lambda e^{x_{k}}} \cdot \frac{e^{x_{k}}}{e^{x_{k}}-\alpha \lambda \widetilde{\Phi}_{k+1}}=\frac{h}{\lambda}, \\
& \frac{1}{1+\alpha^{2} e^{x_{k+1}-x_{k}}} \cdot \frac{e^{x_{k}}-\alpha \lambda \widetilde{\Phi}_{k+1}}{e^{x_{k}}} \cdot \frac{\lambda e^{x_{k}}+\alpha \Psi_{k+1}}{e^{x_{k}}}=\lambda, \\
& \left(1+\alpha(\alpha-h) e^{x_{k+1}-x_{k}}\right) \cdot \frac{e^{x_{k}}}{\lambda e^{x_{k}}+\alpha \Psi_{k+1}} \cdot \frac{\lambda e^{x_{k}}+h \Phi_{k+1}}{e^{x_{k}}-(\alpha-h) \lambda \Phi_{k+1}}=1, \\
& \frac{1}{\alpha e^{x_{k}-x_{k}}-(\alpha-h)} \cdot \frac{e^{x_{k}}-(\alpha-h) \lambda \Phi_{k+1}}{\lambda e^{x_{k}+h \Phi_{k+1}}} \cdot \frac{\Psi_{k}-\alpha \lambda e^{x_{k}}}{\Psi_{k}}=\frac{\lambda}{h}, \\
& \left(1+\alpha^{2} e^{x_{k}-x_{k-1}}\right) \cdot \frac{\Psi_{k}}{\Psi_{k}-\alpha \lambda e^{x_{k}}} \cdot \frac{\widetilde{\Phi}_{k}}{\alpha e^{x_{k}+\lambda \widetilde{\Phi}_{k}}}=\frac{1}{\lambda}, \\
& \frac{1}{1+\alpha(\alpha-h) e^{x_{k}-\widetilde{x}_{k-1}}} \cdot \frac{\alpha e^{x_{k}}+\lambda \widetilde{\Phi}_{k}}{\widetilde{\Phi}_{k}} \cdot \frac{\widetilde{\Psi}_{k}-(\alpha-h) \lambda e^{x_{k}}}{h e^{x_{k}+\lambda \widetilde{\Psi}_{k}}}=1 .
\end{aligned}
$$

Multiplying these equations leads to (15).

System (16). 3D consistent system of quad-equations:

$$
\begin{gathered}
h(X Y+U V)+Y V-(1-h \lambda) X V+h^{2} X U=0, \\
\alpha(X Y+U V)+Y V-(1-\alpha \lambda) X V+\alpha^{2} X U=0, \\
(h-\alpha)(X Y+U V)+(1-\alpha \lambda) Y V-(1-h \lambda) X V+ \\
\quad+h^{2}(1-\alpha \lambda) X U-\alpha^{2}(1-h \lambda) Y U=0 .
\end{gathered}
$$




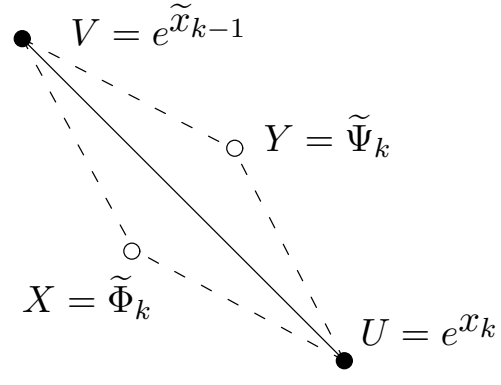

(a) North-western quadrilateral of type III

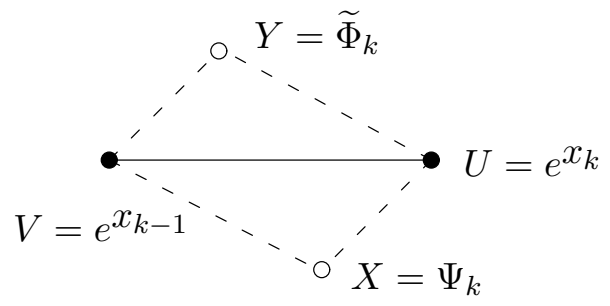

(c) Western quadrilateral of type II

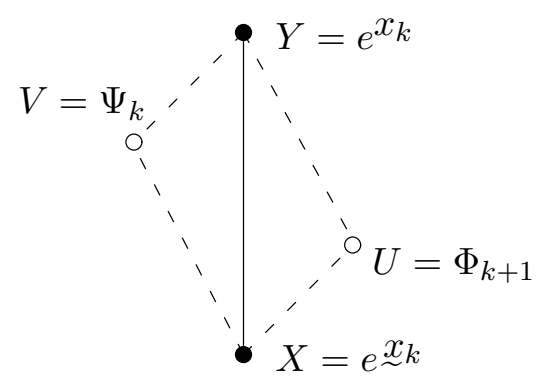

(e) Southern quadrilateral of type I

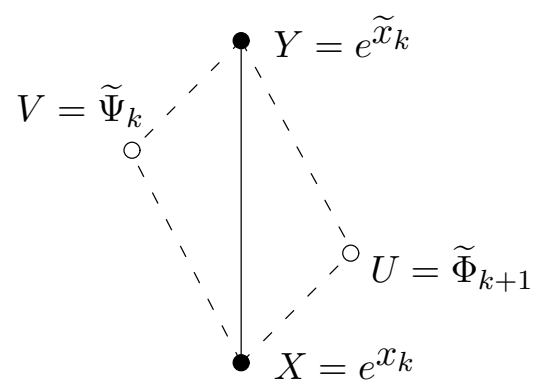

(b) Northern quadrilateral of type I

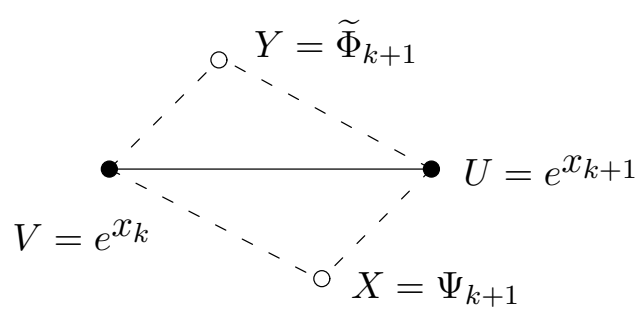

(d) Eastern quadrilateral of type II

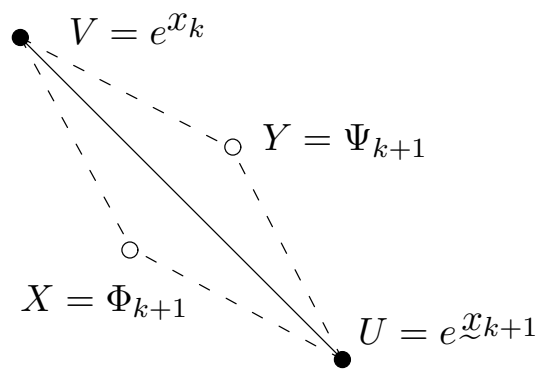

(f) South-eastern quadrilateral of type III

Figure 9: Notation for single quadrilaterals of the dual kagome lattice around the vertex $x_{k}$ 
Three-leg forms of these equations, centered at $x_{k}$ :

$$
\begin{aligned}
& e^{\widetilde{x}_{k}-x_{k}}+\frac{h \widetilde{\Phi}_{k+1}}{e^{x_{k}}}-\frac{(1-h \lambda) \widetilde{\Psi}_{k}}{\widetilde{\Psi}_{k}+h e^{x_{k}}}=0 \\
& -\alpha e^{x_{k+1}-x_{k}}-\frac{\widetilde{\Phi}_{k+1}}{e^{x_{k}}}+\frac{(1-\alpha \lambda) \Psi_{k+1}}{e^{x_{k}}+\alpha \Psi_{k+1}}=0, \\
& \frac{(\alpha-h) e \stackrel{x}{\sim}_{k+1}-x_{k}}{1-h \alpha e e_{k+1}^{x_{k+1}-x_{k}}}-\frac{(1-\alpha \lambda) \Psi_{k+1}}{e^{x_{k}+\alpha \Psi_{k+1}}}+\frac{(1-h \lambda) \Phi_{k+1}}{e^{x_{k}+h \Phi_{k+1}}}=0 \text {, } \\
& -e^{x_{k}-x_{k}}+\frac{(1-h \lambda) e^{x_{k}}}{e^{x_{k}}+h \Phi_{k+1}}-\frac{h e^{x_{k}}}{\Psi_{n}}=0, \\
& \alpha e^{x_{k}-x_{k-1}}+\frac{e^{x_{k}}}{\Psi_{k}}-\frac{(1-\alpha \lambda) e^{x_{k}}}{\widetilde{\Phi}_{k}+\alpha e^{x_{k}}}=0, \\
& -\frac{(\alpha-h) e^{x_{k}-\widetilde{x}_{k-1}}}{1-h \alpha e^{x_{k}-\widetilde{x}_{k-1}}}+\frac{(1-\alpha \lambda) e^{x_{k}}}{\widetilde{\Phi}_{k}+\alpha e^{x_{k}}}-\frac{(1-h \lambda) e^{x_{k}}}{\widetilde{\Psi}_{k}+h e^{x_{k}}}=0 .
\end{aligned}
$$

Adding these equations leads to (16).

System (18). 3D consistent system of quad-equations:

$$
\begin{aligned}
& h \lambda(X Y+U V)-h \lambda^{2} X U-\left(\lambda^{2}+h\right) X V+\lambda^{2} Y V=0, \\
& \alpha \lambda(X Y+U V)-\alpha \lambda^{2} X U-\left(\lambda^{2}+\alpha\right) X V+\lambda^{2} Y V=0, \\
& (h-\alpha) \lambda(X Y+U V)-h\left(\lambda^{2}+\alpha\right) X U-\left(\lambda^{2}+h\right) X V+ \\
& \quad+\alpha\left(\lambda^{2}+h\right) Y U+\left(\lambda^{2}+\alpha\right) Y V=0 .
\end{aligned}
$$

Three-leg forms of these equations, centered at $x_{k}$ :

$$
\begin{aligned}
& \left(e^{\widetilde{x}_{k}-x_{k}}-1\right) \cdot \frac{\lambda \widetilde{\Psi}_{k}+h e^{x_{k}}}{\widetilde{\Psi}_{k}-\lambda e^{x_{k}}} \cdot \frac{e^{x_{k}}}{e^{x_{k}-\lambda \widetilde{\Phi}_{k+1}}}=\frac{h}{\lambda}, \\
& \frac{1}{1+\alpha e^{x_{k+1}-x_{k}}} \cdot \frac{e^{x_{k}}-\lambda \widetilde{\Phi}_{k+1}}{e^{x_{k}}} \cdot \frac{\lambda e^{x_{k}}+\alpha \Psi_{k+1}}{e^{x_{k}-\lambda \Psi_{k+1}}}=\lambda, \\
& \frac{1+\alpha e \sim x_{k+1}-x_{k}}{1+h e^{x} x_{k+1}-x_{k}} \cdot \frac{e^{x_{k}}-\lambda \Psi_{k+1}}{\lambda e^{x_{k}+\alpha \Psi_{k+1}}} \cdot \frac{\lambda e^{x_{k}}+h \Phi_{k+1}}{e^{x_{k}-\lambda \Phi_{k+1}}}=1, \\
& \frac{1}{e^{x_{k}-x_{k}-1}} \cdot \frac{e^{x_{k}}-\lambda \Phi_{k+1}}{\lambda e^{x_{k}+h \Phi_{k+1}}} \cdot \frac{\Psi_{k}-\lambda e^{x_{k}}}{\Psi_{k}}=\frac{\lambda}{h}, \\
& \left(1+\alpha e^{x_{n}-x_{n-1}}\right) \cdot \frac{\Psi_{k}}{\Psi_{k}-\lambda e^{x_{k}}} \cdot \frac{\widetilde{\Phi}_{k}-\lambda e^{x_{k}}}{\lambda \widetilde{\Phi}_{k}+\alpha e^{x_{k}}}=\frac{1}{\lambda}, \\
& \frac{1+h e^{x_{k}-\widetilde{x}_{k-1}}}{1+\alpha e^{x_{k}-\widetilde{x}_{k-1}}} \cdot \frac{\lambda \widetilde{\Phi}_{k}+\alpha e^{x_{k}}}{\widetilde{\Phi}_{k}-\lambda e^{x_{k}}} \cdot \frac{\widetilde{\Psi}_{k}-\lambda e^{x_{k}}}{\lambda \widetilde{\Psi}_{k}+h e^{x_{k}}}=1 .
\end{aligned}
$$

Multiplying these equations leads to (18). 
System (20). 3D consistent system of quad-equations:

$$
\begin{gathered}
h(X Y-X U-Y V+U V)-(1-h \lambda)(X-Y)-h \lambda(U-V)-h \lambda^{2}=0, \\
\alpha(X Y-X U-Y V+U V)-(1-\alpha \lambda)(X-Y)-\alpha \lambda(U-V)-\alpha \lambda^{2}=0, \\
(h-\alpha)(X Y+U V)-h(1-2 \alpha \lambda)(X U+Y V)+\alpha(1-2 h \lambda)(X V+Y U)- \\
\quad-(1-(\alpha+h) \lambda)(X-Y)-(h-\alpha) \lambda(U-V)-(h-\alpha) \lambda^{2}=0 .
\end{gathered}
$$

Three-leg forms of these equations, centered at $x_{k}$ :

$$
\begin{aligned}
& \left(\widetilde{x}_{k}-x_{k}\right) \cdot \frac{1+h\left(x_{k}-\widetilde{\Psi}_{k}-\lambda\right)}{x_{k}-\widetilde{\Psi}_{k}+\lambda} \cdot \frac{1}{x_{k}-\widetilde{\Phi}_{k+1}-\lambda}=-h, \\
& \frac{1}{1+\alpha\left(x_{k+1}-x_{k}\right)} \cdot\left(x_{k}-\widetilde{\Phi}_{k+1}-\lambda\right) \cdot \frac{1-\alpha\left(x_{k}-\Psi_{k+1}+\lambda\right)}{x_{k}-\Psi_{k+1}-\lambda}=1, \\
& \frac{1+\alpha\left(x_{k+1}-x_{k}\right)}{1+h(\underbrace{}_{k+1}-x_{k})} \cdot \frac{x_{k}-\Psi_{k+1}-\lambda}{1-\alpha\left(x_{k}-\Psi_{k+1}+\lambda\right)} \cdot \frac{1-h\left(x_{k}-\Phi_{k+1}+\lambda\right)}{x_{k}-\Phi_{k+1}-\lambda}=1, \\
& \frac{1}{x_{k}-\underline{x}_{k}} \cdot \frac{x_{k}-\Phi_{k+1}-\lambda}{1-h\left(x_{k}-\Phi_{k+1}+\lambda\right)} \cdot\left(x_{k}-\Psi_{k}+\lambda\right)=-\frac{1}{h}, \\
& \left(1+\alpha\left(x_{k}-x_{k-1}\right)\right) \cdot \frac{1}{x_{k}-\Psi_{k}+\lambda} \cdot \frac{x_{k}-\widetilde{\Phi}_{k}+\lambda}{1+\alpha\left(x_{k}-\widetilde{\Phi}_{k}-\lambda\right)}=1, \\
& \frac{1+h\left(x_{k}-\widetilde{x}_{k-1}\right)}{1+\alpha\left(x_{k}-\widetilde{x}_{k-1}\right)} \cdot \frac{1+\alpha\left(x_{k}-\widetilde{\Phi}_{k}-\lambda\right)}{x_{k}-\widetilde{\Phi}_{k}+\lambda} \cdot \frac{x_{k}-\widetilde{\Psi}_{k}+\lambda}{1+h\left(x_{k}-\widetilde{\Psi}_{k}-\lambda\right)}=1 .
\end{aligned}
$$

Multiplying these equations leads to (20).

\section{The master system}

It turns out that all the 3D consistent systems of quad-equations leading to nonsymmetric discrete relativistic Toda systems (those given above and those omitted for the space reasons), as well as the systems Q1 and $\mathrm{Q} 3_{\delta=0}$ leading to the symmetric discrete relativistic Toda systems are particular or limiting cases of one multi-parametric system. Thus this latter system can be seen as the master one behind the whole theory of the relativistic Toda systems of the type (14) (with discrete time) and (11) (with continuous time).

Master system of quad-equations:

$$
\begin{aligned}
& (\delta-\beta \gamma) \lambda(X Y+U V)+\beta \delta\left(\lambda^{2}-\gamma\right) X U+\left(\beta \lambda^{2}-\delta\right) X V+ \\
& \quad+\gamma\left(\beta \lambda^{2}-\delta\right) Y U+\left(\lambda^{2}-\gamma\right) Y V=0 \\
& (\eta-\gamma \epsilon) \lambda(X Y+U V)+\left(\lambda^{2}-\gamma\right) X U+\left(\epsilon \lambda^{2}-\eta\right) X V+ \\
& \quad+\gamma\left(\epsilon \lambda^{2}-\eta\right) Y U+\epsilon \eta\left(\lambda^{2}-\gamma\right) Y V=0 \\
& (\beta \eta-\delta \epsilon) \lambda(X Y+U V)-\beta \delta\left(\epsilon \lambda^{2}-\eta\right) X U+\epsilon \eta\left(\beta \lambda^{2}-\delta\right) X V+ \\
& \quad+\left(\beta \lambda^{2}-\delta\right) Y U-\left(\epsilon \lambda^{2}-\eta\right) Y V=0 .
\end{aligned}
$$


Three-leg forms of these equations, centered at $x_{k}$ :

$$
\begin{aligned}
& \frac{e^{\widetilde{x}_{k}}+\beta e^{x_{k}}}{\gamma e^{\widetilde{x}_{k}}+\delta e^{x_{k}}} \cdot \frac{\delta e^{x_{k}}+\lambda \widetilde{\Psi}_{k}}{\beta \lambda e^{x_{k}}+\widetilde{\Psi}_{k}} \cdot \frac{\lambda e^{x_{k}}-\gamma \widetilde{\Phi}_{k+1}}{e^{x_{k}}-\lambda \widetilde{\Phi}_{k+1}}=1, \\
& \frac{\gamma e^{x_{k+1}}+\eta e^{x_{k}}}{e^{x_{k+1}}+\epsilon e^{x_{k}}} \cdot \frac{e^{x_{k}}-\lambda \widetilde{\Phi}_{k+1}}{\lambda e^{x_{k}}-\gamma \widetilde{\Phi}_{k+1}} \cdot \frac{\epsilon \lambda e^{x_{k}}+\Psi_{k+1}}{\eta e^{x_{k}}+\lambda \Psi_{k+1}}=1 \text {, } \\
& \frac{\beta e e_{k+1}^{x_{k}}-\epsilon e^{x_{k}}}{\delta e \underset{\sim}{\sim} k+1-\eta e^{x_{k}}} \cdot \frac{\eta e^{x_{k}}+\lambda \Psi_{k+1}}{\epsilon \lambda e^{x_{k}}+\Psi_{k+1}} \cdot \frac{\lambda e^{x_{k}}+\delta \Phi_{k+1}}{e^{x_{k}}+\beta \lambda \Phi_{k+1}}=1, \\
& \frac{\gamma e^{x_{k}}+\delta e \stackrel{x_{k}}{\sim}}{e^{x_{k}}+\beta e^{x}} \cdot \frac{e^{x_{k}}+\beta \lambda \Phi_{k+1}}{\lambda e^{x_{k}}+\delta \Phi_{k+1}} \cdot \frac{\lambda e^{x_{k}}-\Psi_{k}}{\gamma e^{x_{k}}-\lambda \Psi_{k}}=1, \\
& \frac{e^{x_{k}}+\epsilon e^{x_{k-1}}}{\gamma e^{x_{k}+\eta e^{x_{k-1}}}} \cdot \frac{\gamma e^{x_{k}}-\lambda \Psi_{k}}{\lambda e^{x_{k}}-\Psi_{k}} \cdot \frac{\lambda e^{x_{k}}+\eta \widetilde{\Phi}_{k}}{e^{x_{k}+\epsilon \lambda \widetilde{\Phi}_{k}}}=1, \\
& \frac{\delta e^{x_{k}}-\eta e^{\widetilde{x}_{k-1}}}{\beta e^{x_{k}}-\epsilon e^{\widetilde{x}_{k-1}}} \cdot \frac{e^{x_{k}}+\epsilon \lambda \widetilde{\Phi}_{k}}{\lambda e^{x_{k}}+\eta \widetilde{\Phi}_{k}} \cdot \frac{\beta \lambda e^{x_{k}}+\widetilde{\Psi}_{k}}{\delta e^{x_{k}}+\lambda \widetilde{\Psi}_{k}}=1 .
\end{aligned}
$$

Multiplying these three-leg forms leads to the following general equation of the discrete relativistic Toda type:

$$
\begin{aligned}
& \frac{e^{\widetilde{x}_{k}-x_{k}}+\beta}{\gamma e^{\widetilde{x}_{k}-x_{k}+\delta}} \cdot \frac{\gamma e^{x_{k}-x_{k}}+\delta}{e^{x_{k}-x_{k}+\beta}} \\
& \cdot \frac{\gamma e^{x_{k+1}-x_{k}}+\eta}{e^{x_{k+1}-x_{k}}+\epsilon} \cdot \frac{e^{x_{k}-x_{k-1}+\epsilon}}{\gamma e^{x_{k}-x_{k-1}+\eta}} \\
& \cdot \frac{\beta e^{x_{k+1}-x_{k}-\epsilon}}{\delta e x_{k+1}-x_{k}-\eta} \cdot \frac{\delta e^{x_{k}-\widetilde{x}_{k-1}-\eta}}{\beta e^{x_{k}-\widetilde{x}_{k-1}-\epsilon}}=1 .
\end{aligned}
$$

This equation has five parameters $\beta, \gamma, \delta, \epsilon, \eta$. Actually there are only four, because of homogeneity: if $\gamma \neq 0$, we can set $\gamma=1$ by replacing $\delta, \eta$ through $\delta / \gamma, \eta / \gamma$, respectively. Moreover, we could eliminate two further parameters by shifts of the form $x_{k}(t) \rightarrow$ $x_{k}(t)+A k+B t$, which keep the form of the equation invariant.

It is not difficult to find out the special values of parameters which lead to all the discrete relativistic Toda type equations listed in Section 3. In particular, the value $\gamma=1$ leads to the most general symmetric equation (22), with further degenerations to (23), (24), (25). The value $\gamma=0$ is of the primary interest for the aims of the present paper, since it leads to

$$
\frac{e^{\widetilde{x}_{k}-x_{k}}+\beta}{e^{x_{k}-x_{k}+\beta}} \cdot \frac{e^{x_{k}-x_{k-1}}+\epsilon}{e^{x_{k+1}-x_{k}+\epsilon}} \cdot \frac{\beta e^{x_{k+1}-x_{k}}-\epsilon}{\delta e_{\sim}^{x_{k+1}-x_{k}-\eta}} \cdot \frac{\delta e^{x_{k}-\widetilde{x}_{k-1}}-\eta}{\beta e^{x_{k}-\widetilde{x}_{k-1}-\epsilon}}=1,
$$

which happens to encapsulate all the non-symmetric equations. For instance:

- System (15) appears from (38) with the choice $\eta=\infty, \beta=(h-\alpha) / \alpha$ and $\epsilon=1 / \alpha^{2}$. In the quad-equations it is convenient to set $\delta=\alpha h$ and to re-scale $\lambda \rightsquigarrow \alpha \lambda$. 
- System (18) appears from (38) with the choice $\beta=\eta=-1, \delta=h$ and $\epsilon=1 / \alpha$.

- One gets from (38) to the additive equation (16) in two steps. On the first step, one starts with the parameter values $\beta=(h-\theta) / \theta, \eta=\theta /(\alpha-\theta), \delta=h \theta$, $\epsilon=1 /(\alpha \theta)$, which leads to a remarkable equation introduced in [18]:

$$
\begin{aligned}
& \frac{1+\theta h^{-1}\left(e^{\widetilde{x}_{k}-x_{k}}-1\right)}{1+\theta h^{-1}\left(e^{x_{k}-x_{k}}-1\right)}= \\
& \quad=\frac{1+\theta \alpha e^{x_{k+1}-x_{k}}}{1+\theta \alpha e^{x_{k}-x_{k-1}}} \cdot \frac{1+h(\theta-\alpha) e^{x_{k+1}-x_{k}}}{1+\alpha(\theta-h) e^{x_{k+1}-x_{k}}} \cdot \frac{1+\alpha(\theta-h) e^{x_{k}-\widetilde{x}_{k-1}}}{1+h(\theta-\alpha) e^{x_{k}-\widetilde{x}_{k-1}}}
\end{aligned}
$$

This equation interpolates between (15) (corresponding to $\theta=\alpha$ ) and (16) (which corresponds to $\theta=0)$. In the corresponding quad-equations it is convenient to re-scale $\lambda \rightsquigarrow \theta \lambda$. We remark that the last equation is a time discretization of

$$
\begin{aligned}
\ddot{x}_{k}= & \left(1+\theta \dot{x}_{k}\right)\left(\left(1+\alpha \dot{x}_{k+1}\right) \frac{e^{x_{k+1}-x_{k}}}{1+\theta \alpha e^{x_{k+1}-x_{k}}}-\left(1+\alpha \dot{x}_{k-1}\right) \frac{e^{x_{k}-x_{k-1}}}{1+\theta \alpha e^{x_{k}-x_{k-1}}}\right. \\
& \left.+\alpha(\theta-\alpha) \frac{e^{2\left(x_{k+1}-x_{k}\right)}}{1+\theta \alpha e^{x_{k+1}-x_{k}}}-\alpha(\theta-\alpha) \frac{e^{2\left(x_{k}-x_{k-1}\right)}}{1+\theta \alpha e^{x_{k}-x_{k-1}}}\right),
\end{aligned}
$$

which in turn interpolates between the continuous time equations (21) (for $\theta=\alpha$ ) and (3) (for $\theta=0$ ). On the second step, one performs in equation (*) the limit $\theta \rightarrow 0$. In this limit one should also re-scale the auxiliary variables according to $\Psi \rightsquigarrow \lambda \Psi, \Phi \rightsquigarrow \Phi / \lambda$, and $\lambda \rightsquigarrow 1+\theta \lambda / 2$.

- To transform (38) to the rational equation (20) one makes the change of variables

$$
x \rightsquigarrow \kappa x,
$$

accompanied by the change of parameters

$$
\beta \rightsquigarrow-1+\kappa \beta, \quad \delta \rightsquigarrow-1+\kappa \delta, \quad \epsilon \rightsquigarrow-1+\kappa \epsilon, \quad \eta \rightsquigarrow-1+\kappa \eta .
$$

Sending $\kappa \rightarrow 0$, one arrives at

$$
\frac{\widetilde{x}_{k}-x_{k}+\beta}{x_{k}-\underbrace{}_{k}+\beta} \cdot \frac{x_{k}-x_{k-1}+\epsilon}{x_{k+1}-x_{k}+\epsilon} \cdot \frac{x_{k+1}-x_{k}-\beta+\epsilon}{\underbrace{}_{k+1}-x_{k}-\delta+\eta} \cdot \frac{x_{k}-\widetilde{x}_{k-1}-\delta+\eta}{x_{k}-\widetilde{x}_{k-1}-\beta+\epsilon}=1 .
$$

Equation (20) corresponds to the choice

$$
\beta=\eta=0, \quad \delta=-1 / h, \quad \epsilon=1 / \alpha .
$$




\section{Zero curvature representations}

The construction of discrete Toda type systems on graphs from systems of quad-equations allows one to find, in an algorithmic way, discrete zero curvature representations for the former. Indeed, each quad-equation can be viewed as a Möbius transformation of the field at one white vertex of a quad into the field at the other white vertex, with the coefficients dependent on the fields at the both black vertices. The $S L(2, \mathbb{C})$ matrices representing these Möbius transformations play then the role of transition matrices across the edges connecting the black vertices. The property (28) is satisfied automatically, by construction.

Specializing this construction to the case of the regular triangular lattice (see Figure 7), we denote by $L_{k}$ the transition matrix from $\Psi_{k}$ to $\Psi_{k+1}$, and by $V_{k}$ the transition matrix from $\Psi_{k}$ to $\widetilde{\Psi}_{k}$. In this notation, the discrete zero representation reads:

$$
\widetilde{L}_{k} V_{k}=V_{k+1} L_{k}
$$

both parts representing the transition from $\Psi_{k}$ to $\widetilde{\Psi}_{k+1}$ along two different paths. It is clear that $L_{k}$ is the product of two matrices, the first corresponding to the transition from $\Psi_{k}$ to $\Phi_{k+1}$ across the edge $\left[x_{k},{\underset{\sim}{x}}_{k}\right]$, and the second corresponding to the transition from $\Phi_{k+1}$ to $\Psi_{k+1}$ across the edge $\left[x_{k}, x_{k+1}\right]$, so that

$$
L_{k}=L\left(x_{k}, \underset{\sim}{x_{k}}, \underset{\sim}{x_{k+1}} ; \lambda\right) .
$$

Similarly, $V_{k}$ can be represented as the product of two matrices, the first corresponding to the transition from $\Psi_{k}$ to $\widetilde{\Phi}_{k}$ across the edge $\left[x_{k}, x_{k-1}\right]$, and the second corresponding to the transition from $\widetilde{\Phi}_{k}$ to $\widetilde{\Psi}_{k}$ across the edge $\left[x_{k}, \widetilde{x}_{k-1}\right]$, so that

$$
V_{k}=V\left(x_{k}, x_{k-1}, \widetilde{x}_{k-1} ; \lambda\right) .
$$

The matrices $L_{k}, V_{k}$ for a given discrete relativistic Toda type equation can be computed in a straightforward way, as soon as the generating system of quad-equations is known. Theorem 2 provides us with the means for this goal.

The resulting zero curvature representations possess an additional remarkable property. It is well known (see, e.g., 4], [7]) that the discrete relativistic Toda type equations possess a Lagrangian (variational) interpretation with a discrete Lagrange function

$$
\mathcal{L}(x, \underset{\sim}{x})=\sum_{k \in \mathbb{Z}}\left(\Lambda_{1}(x_{k}-\underbrace{}_{k})-\Lambda_{2}(\underbrace{x_{k+1}}_{k+1}-\underbrace{x}_{k})-\Lambda_{3}(\underbrace{x_{k+1}}_{k+1}-x_{k})\right) .
$$

Here $\Lambda_{1}, \Lambda_{2}, \Lambda_{3}$ are antiderivatives of the functions $F, G, H$ in the general equation (14). The canonically conjugate momenta and the Lagrangian form of equations of motion are given by

$$
p_{k}=\frac{\partial \mathcal{L}(x, \underset{x}{)})}{\partial x_{k}}=-\frac{\partial \mathcal{L}(\widetilde{x}, x)}{\partial x_{k}},
$$

which specializes in our case to

$$
\begin{aligned}
p_{k} & =F(x_{k}-\underbrace{}_{k})+H(\underbrace{}_{k+1}-x_{k}) \\
& =F\left(\widetilde{x}_{k}-x_{k}\right)+H\left(x_{k}-\widetilde{x}_{k-1}\right)-G\left(x_{k+1}-x_{k}\right)+G\left(x_{k}-x_{k-1}\right) .
\end{aligned}
$$


Theorem 3. For all discrete relativistic Toda type systems, the transition matrix $L_{k}$ from equation (40) is local, when expressed in terms of canonically conjugate variables:

$$
L_{k}=L\left(x_{k}, p_{k} ; \lambda\right) \text {. }
$$

Moreover, as a matter of fact, the matrix $L_{k}$ does not depend on the time discretization parameter $h$, so that the corresponding Lagrangian maps $(x, p) \mapsto(\widetilde{x}, \widetilde{p})$ belong to the same integrable hierarchies as their respective continuous time Hamiltonian counterparts. In other words, these Lagrangian maps serve as Bäcklund transformations for the respective Hamiltonian flows, the Bäcklund parameter being the time step $h$.

Proof of this theorem is obtained again via direct computations on the case-by-case basis. For all cases where the local discrete zero curvature representation was known (those cases are listed in [18]) the results obtained from the system of quad-equations coincide with the previously available ones. Therefore, we illustrate the claims of the theorem with the case where the local discrete zero curvature representation was not known previously, namely with the rational systems (20), (21). It is useful to keep in mind that these two systems come as two elementary flows (a positive and a negative ones) of the same hierarchy [18].

Equation (20). The Lagrangian form reads:

$$
\begin{aligned}
h e^{p_{k}} & =\left(x_{k}-\underline{x}_{k}\right) \cdot \frac{1+h\left({\underset{x}{k+1}}_{k+x_{k}}\right)}{1+\alpha(\underbrace{}_{k+1}-x_{k})} \\
& =\left(\widetilde{x}_{k}-x_{k}\right) \cdot \frac{1+\alpha\left(x_{k}-x_{k-1}\right)}{1+\alpha\left(x_{k+1}-x_{k}\right)} \cdot \frac{1+h\left(x_{k}-\widetilde{x}_{k-1}\right)}{1+\alpha\left(x_{k}-\widetilde{x}_{k-1}\right)} .
\end{aligned}
$$

The transition matrices of the zero curvature representation of this map read:

$$
L_{k}=\left(\begin{array}{cc}
-\lambda+x_{k} & \lambda^{2}+\lambda \alpha e^{p_{k}}-\left(x_{k}-\alpha e^{p_{k}}\right) x_{k}-e^{p_{k}} \\
1 & -\lambda-x_{k}+\alpha e^{p_{k}}
\end{array}\right)
$$

and

$$
V_{k}=I+h\left(\begin{array}{cc}
-\lambda+x_{k} & \lambda^{2}+\lambda\left(x_{k}-\widetilde{x}_{k-1}+\alpha e^{\widetilde{p}_{k-1}}\right)-\left(\widetilde{x}_{k-1}-\alpha e^{\widetilde{p}_{k-1}}\right) x_{k} \\
1 & -\lambda-\widetilde{x}_{k-1}+\alpha e^{\widetilde{p}_{k-1}}
\end{array}\right) .
$$

Note that in the limit $h \rightarrow 0$ one obtains a zero curvature representation (7) for the Hamiltonian form of equation (8) with the same matrix $L_{k}$ as in (48) and with the matrix

$$
M_{k}=\left(\begin{array}{cc}
-\lambda+x_{k} & \lambda^{2}+\lambda\left(x_{k}-x_{k-1}+\alpha e^{p_{k-1}}\right)-\left(x_{k-1}-\alpha e^{p_{k-1}}\right) x_{k} \\
1 & -\lambda-x_{k-1}+\alpha e^{p_{k-1}}
\end{array}\right) .
$$

To the best of our knowledge, this result (and even its non-relativistic particular case $\alpha=0$ ) was previously unknown. 
Equation (21). The Lagrangian form reads:

$$
\begin{aligned}
h e^{p_{k}} & =\frac{\widetilde{x}_{k}-x_{k}}{1+\alpha(\alpha+h) h^{-1}\left(\widetilde{x}_{k}-x_{k}\right)} \cdot\left(1+(\alpha+h)\left(x_{k}-\widetilde{x}_{k-1}\right)\right) \\
& =\frac{x_{k}-\underbrace{}_{k}}{1+\alpha(\alpha+h) h^{-1}(x_{k}-\underbrace{}_{k})} \cdot \frac{1+\alpha\left(x_{k+1}-x_{k}\right)}{1+\alpha\left(x_{k}-x_{k-1}\right)} \cdot\left(1+(\alpha+h)(\underbrace{}_{k+1}-x_{k})\right) .
\end{aligned}
$$

This Lagrangian map admits a discrete zero curvature representation

$$
W_{k+1} \widetilde{L}_{k}=L_{k} W_{k}
$$

with the same transition matrix $L_{k}$ as in (48) and with

$$
\begin{aligned}
W_{k}= & I-\frac{h}{(1-2 \alpha \lambda)\left(1+(\alpha+h)\left(x_{k}-\widetilde{x}_{k-1}-\alpha e p_{k}\right)\right)} \\
& \times\left(\begin{array}{cc}
\lambda+x_{k}-\alpha e^{p_{k}} & \lambda^{2}+\lambda\left(x_{k}-\widetilde{x}_{k-1}-\alpha e^{p_{k}}\right)-\left(x_{k}-\alpha e^{p_{k}}\right) x_{k-1} \\
1 & \lambda-\widetilde{x}_{k-1}
\end{array}\right) .
\end{aligned}
$$

Again, in the limit $h \rightarrow 0$ one obtains a zero curvature representation (7) for the Hamiltonian form of equation (9) with the same matrix $L_{k}$ as in (48) and with the matrix

$$
\begin{aligned}
M_{k}= & \frac{1}{(1-2 \alpha \lambda)\left(1+\alpha\left(x_{k}-x_{k-1}-\alpha e^{p_{k}}\right)\right)} \\
& \times\left(\begin{array}{cc}
\lambda+x_{k}-\alpha e^{p_{k}} & \lambda^{2}+\lambda\left(x_{k}-x_{k-1}-\alpha e^{p_{k}}\right)-\left(x_{k}-\alpha e^{p_{k}}\right) x_{k-1} \\
1 & \lambda-x_{k-1}
\end{array}\right) .
\end{aligned}
$$

Also these results seem to be previously unknown, even in the continuous time case.

\section{Conclusions}

In this paper, we clarified the origin of all non-symmetric discrete equations of the relativistic Toda type from 3D consistent systems of quad-equations. Unlike the symmetric case, the three coordinate planes carry here three different quad-equations, so that a more general understanding of the 3D consistency than usual is required. Note that this more general concept has already been discussed and laid into the basis of a classification procedure in [5], see also examples discussed in [8]. However, the classification only has been performed for the so called systems of type Q in [5] (all edge biquadratics nondegenerate, see the original paper for details). Examples which arose in the present work demonstrate that also the systems of type $\mathrm{H}$ (with some of the edge biquadratics being degenerate) are of a considerable importance, which calls for a complete classification of this case, as well. We plan to turn to this tedious task in our future work. 


\section{References}

[1] V.E. Adler, Legendre transformations on the triangular lattice, Funct. Anal. Appl., 34 (1999), pp. 1-9.

[2] - On the structure of the Bäcklund transformations for the relativistic lattices, J. Nonlin. Math. Phys. 7 (2000), pp. 34-56.

[3] — Discrete equations on planar graphs, J. Phys. A: Math. Gen. 34 (2001), pp. 10453-10460.

[4] V.E. Adler, A.I. Bobenko, Yu.B. Suris, Classification of integrable equations on quad-graphs. The consistency approach, Comm. Math. Phys. 233 (2003), pp. 513543.

[5] V.E. Adler, A.I. Bobenko, Yu.B. Suris, Discrete nonlinear hyperbolic equations. Classification of integrable cases, Funct. Anal. Appl. 43 (2009), pp. 3-17.

[6] V.E. Adler and A.B. Shabat, Generalized Legendre transformations, Theor. Math. Phys. 112 (1997), pp. 935-948.

[7] V.E. Adler, Yu.B. Suris, Q4: integrable master equation related to an elliptic curve, Intern. Math. Research Notices, Nr. 47 (2004), pp. 2523-2553.

[8] J. Atkinson, Bäcklund transformations for integrable lattice equations, J. Phys. A: Math. Theor. 41 (2008) 135202, 8 pp.

[9] A.I. Bobenko, Ch. Mercat, Yu.B. Suris, Linear and nonlinear theories of discrete analytic functions. Integrable structure and isomonodromic Green's function, J. Reine Angew. Math., 583 (2005), pp. 117-161.

[10] A.I. Bobenko, Yu.B. Suris. Integrable systems on quad-graphs, Intern. Math. Research Notices, Nr. 11 (2002), pp. 573-611.

[11] - Discrete Differential Geometry. Integrable Structure, Graduate Studies in Mathematics, Vol. 98. AMS, Providence, 2008.

[12] R. Boll, Embedding of Non-Symmetric Discrete Toda Systems in Multidimensional Lattices, Diploma thesis, TU München, 2009.

[13] F. Nijhoff, Lax pair for the Adler (lattice Krichever-Novikov) system, Phys. Lett. A 297 (2002), pp. 49-58.

[14] S.N.M. Ruijsenaars, Relativistic Toda systems, Commun. Math. Phys. 133 (1990), pp. 217-247.

[15] Yu.B. Suris, A discrete-time relativistic Toda lattice, J. Phys. A: Math. and Gen. 29 (1996), pp. 451-465. 
[16] - New integrable systems related to the relativistic Toda lattice, J. Phys. A: Math. and Gen. 30 (1997), pp. 1745-1761.

[17] - R-matrix hierarchies, integrable lattice systems, and their integrable discretizations, in Symmetries and Integrability of Difference Equations, P.Clarkson and F.Nijhoff, eds, Cambridge Univ. Press, 1999, pp. 79-94.

[18] - The Problem of Integrable Discretization. Hamiltonian Approach, Progress in Mathematics Vol. 219, Birkhäuser, Basel, 2003. 